\title{
BOUNDARY FITTING PROBLEMS ASSOCIATED WITH CORONAL MAGNETIC MODELS
}

\author{
KENNETH H. SCHATTEN \\ Victoria University, Wellington, New Zealand
}

\begin{abstract}
The calculation of coronal magnetic fields was first suggested by Gold (1958). Altschuler and Newkirk (1969) and Newkirk et al. (1968) used a Legendre polynomial fit to the photospheric observations of magnetic fields whereas Schatten (1968) with Wilcox and Ness (Schatten et al., 1969) use a magnetic monopole fit, first incorporated by Schmidt (1964).
\end{abstract}

Altschuler and Newkirk berate the monopole fit as not being 'mathematically valid'. Although physical reasons are not provided in their paper, the only physical reason which may be difficult for some to understand is the following. In the monopole fit one uses $\nabla \cdot B=4 \pi \varrho_{m}$ where $\varrho_{m}$ is a magnetic monopole charge density necessary to fit the photospheric observations. The non-zero divergence of the magnetic field is mathematically valid, but not physically valid, if one were to use the magnetic field solution in the region where the monopoles were located. As the monopoles are only embedded beneath the photosphere (where the solution is not calculated) the solution obtained by those authors who use this technique is valid in the regions of space where the field has been calculated (the corona). The remaining question is which technique fits boundary conditions (in the photosphere) more accurately. As no one has obtained a method of objective comparison with observations, the only test one has available, is to see which technique best fits the observations.

As a test to measure the 'quality of fit' of these two procedures, I have calculated the mean square difference between a calculated magnetic field (at 1.05 solar radii a small distance above the photosphere) and the raw photospheric observations. A value of 0.0 would mean a perfect fit was obtained. The values were normalized so that a quality of fit equal to 1.0 would indicate the difference between the raw data and a constant value of zero. Using the Legendre polynomial technique with $N=9$ (the same value used by Altschuler and Newkirk (1969)) a quality of fit equal to 0.77 was obtained for the November 12, 1966 solar eclipse photospheric data and a value of 0.36 was obtained using a monopole fit with a comparable grid spacing. Similar values were obtained with data at other eclipse periods.

The closer fit obtained by the monopole technique appears, at first, surprising. However when one looks at the behaviour of the photospheric field, it is not. The photospheric field is 'clumpy', there are weak fields and strong fields. The monopole technique can fit combinations of these fields equally well. The Legendre polynomial technique attempts the fit of a 'smooth' function to this clumpy data. Hence a poor fit can result with this technique. Furthermore there is no Legendre index $N$, however large, which will adequately describe a fit to a point source. If the photospheric 
field were 'smoother' the Legendre technique would be better, however it is not. The problem of field lines 'going through the Sun' is solved through the use of a Green's function solution in my work.

It is interesting to note that the same problem occurs in gravitational anomalies associated with mass concentrations on the moon (mascons). The problem is resolved in an identical fashion. It is found that these mascons can only be described (gravitationally) by the inclusion of point sources in the gravitational field. Thus the Sun appears in a magnetic sense, similar to the Moon in a gravitational sense - the effect of active regions being identical to that of mascons.

\section{Acknowledgements}

I am grateful to Dave Howell, who helped with the calculations and for the benefit of working with R. Howard, J. Wilcox and N. F. Ness. I thank R. Howard of the Hale Observatories and Carnegie Institute for the use of his photospheric field data obtained in a program in part by the Office of Naval Research under contract NR 013-023, N000 14-66-C-0239.

\section{References}

Altschuler, M. D. and Newkirk, G., Jr.: 1969, Solar Phys. 9, 131.

Gold, T. : 1958, in B. Lehnert (ed.), 'Electromagnetic Phenomena in Cosmical Physics', IAU Symp. 6, 275.

Newkirk, G., Jr., Altschuler, M. D., and Harvey, T. W.: 1968, in K. O. Kiepenheuer (ed.), 'Structure and

Development of Solar Active Regions', IAU Symp. 35, 379.

Schatten, K. H.: 1968, Ph.D. Thesis, Univ. of Calif. Berkeley.

Schatten, K. H., Wilcox, J. M., and Ness, N. F.: 1969, Solar Phys. 6, 442.

Schmidt, H. O.: 1964, in W. Hess (ed.), AAS-NASA Symposium on Physics of Solar Flares, NASA SP-50, p. 107.

\section{DISCUSSION}

Schmidt: I proposed the monopole method only for fitting the surface field on a plane such as might be applied to active regions. Loop prominences represent the only case where the force-free field method has given a demonstrably correct picture. This can be understood because during the flare the energy has been released and the field relaxed to the potential configuration.

Schatten: You say all large-scale current-free models are not appropriate. Thus you have the same criticism of the Legendre technique as you have with monopole technique. Both techniques make the same assumptions outside the Sun.

Altschuler: The problem is that we cannot use the line-of-sight magnetic measurements to choose values for monopoles in a spherical surface. On a spherical surface, any monopole produces a contribution on the other side of the Sun. Consequently, the total distribution of monopoles you finally get is not consistent with the originally given boundary condition.

Schatten's statistical test is also misleading; when he compares the calculated field values at $r=1.05 R_{\odot}$ with the raw data, he is only showing that the field values he chooses at the surface can be regurgitated immediately above the surface. The problem, however, is to obtain the large-scale pattern of magnetic field directions. In the Legendre polynomial method we have limited resolution at the surface with principal index $N=9$, but higher in the corona only the lowest harmonics survive anyway, so the crucial matter is not high resolution but the proper magnetic field directions on the large-scale. There is no difficulty in applying the Legendre polynomial method with a much larger principal index than $N=9$, but we have not done so because, as Dr Schmidt points out, there are only limited applications for the potential field calculation. 
Schatten: I used only magnetic monopole sources from regions less than about $30-40$ deg removed from the point of field computation. This, then, prevents magnetic field from going through the Sun. Effectively, I used a Green's function $\bar{G} \approx\left(\hat{r}-\hat{r}_{m}\right) / r_{m}^{2}$ for $\theta<30-40^{\circ}$ and $\bar{G} \approx 0$ for $\theta>30-40^{\circ}$, where $\theta$ is the angle between the source and the point of field computation. Thus, the magnetic field from a point on one side of the Sun does not produce a significant flux on the opposite side of the Sun as you suggest. I think the way to go in future analyses is in the direction of Pneuman and Kopp, that is, one should consider the interaction of the solar wind with the magnetic field. 